\title{
Assessment of glomerular morphological patterns by deep learning algorithms
}

\author{
Cleo-Aron Weis ${ }^{1}$ (D) Jan Niklas Bindzus ${ }^{1} \cdot$ Jonas Voigt $^{1} \cdot$ Marlen Runz $^{1,2} \cdot$ Svetlana Hertjens $^{3} \cdot$ Matthias M. Gaida $^{4}$. \\ Zoran V. Popovic ${ }^{1}$. Stefan Porubsky ${ }^{4}$
}

Accepted: 30 November 2021 / Published online: 4 January 2022

(c) The Author(s) 2022

\begin{abstract}
Background Compilation of different morphological lesion signatures is characteristic of renal pathology. Previous studies have documented the potential value of artificial intelligence (AI) in recognizing relatively clear-cut glomerular structures and patterns, such as segmental or global sclerosis or mesangial hypercellularity. This study aimed to test the capacity of deep learning algorithms to recognize complex glomerular structural changes that reflect common diagnostic dilemmas in nephropathology.

Methods For this purpose, we defined nine classes of glomerular morphological patterns and trained twelve convolutional neuronal network $(\mathrm{CNN})$ models on these. The two-step training process was done on a first dataset defined by an expert nephropathologist (12,253 images) and a second consensus dataset (11,142 images) defined by three experts in the field.

Results The efficacy of CNN training was evaluated using another set with 180 consensus images, showing convincingly good classification results (kappa-values 0.838-0.938).

Furthermore, we elucidated the image areas decisive for CNN-based decision making by class activation maps. Finally, we demonstrated that the algorithm could decipher glomerular disease patterns coinciding in a single glomerulus (e.g. necrosis along with mesangial and endocapillary hypercellularity).

Conclusions In summary, our model, focusing on glomerular lesions detectable by conventional microscopy, is the first sui generis to deploy deep learning as a reliable and promising tool in recognition of even discrete and/or overlapping morphological changes. Our results provide a stimulus for ongoing projects that integrate further input levels next to morphology (such as immunohistochemistry, electron microscopy, and clinical information) to develop a novel tool applicable for routine diagnostic nephropathology.
\end{abstract}

Keywords Machine learning $\cdot \mathrm{CNN} \cdot$ Classification $\cdot$ Glomerular change pattern

Zoran V. Popovic and Stefan Porubsky contributed equally to this work.

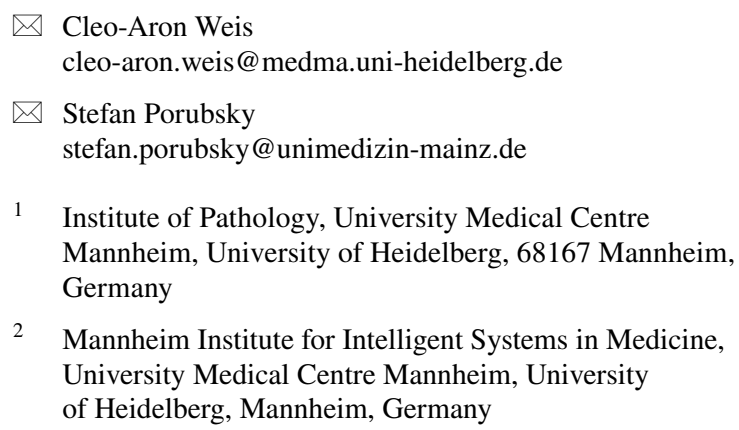

2 Mannheim Institute for Intelligent Systems in Medicine, University Medical Centre Mannheim, University of Heidelberg, Mannheim, Germany

3 Institute of Medical Statistics and Biometry, Medical Faculty Mannheim, University of Heidelberg, Mannheim, Germany

4 Institute of Pathology, University Medical Center of the Johannes Gutenberg University Mainz, Langenbeckstrasse 1, 55131 Mainz, Germany 


\section{Introduction}

Artificial intelligence is an umbrella term encompassing techniques that enable machines to mimic human intelligence. Machine learning is a subset of artificial intelligence that refers to algorithms capable of learning without being explicitly programmed. Here, amongst others, so-called convolutional neural networks (CNNs) are implemented to independently 'comprehend' characteristic features in large image datasets. Recent substantial progress in image visualization and computation has made CNN-based machine learning an affordable approach that is of utmost interest for diagnostic image-based disciplines, particularly radiology and pathology [1-3]. Due to the plethora of sometimes overlapping clinical and histological disease patterns, nephropathology represents a challenging discipline. This holds true also for the evaluation of histology sections from experiments with kidney diseases in animal models. Here, maybe even more than in human samples, a higher degree of automation and standardization is needed. Algorithms for segmentation of healthy kidney parenchyma have been previously successfully developed $[4,5]$. Similarly, computational morphologic analyses of diabetic nephropathy, mesangial proliferation, and IgA-Nephropathy pattern have been published [6-9]. However, until now, no CNN-based approach that simultaneously deals with various glomerular lesions and that can discern them from unaffected glomeruli has been reported.

A wide variety of glomerular lesions linked to specific kidney diseases have been well characterized [10]. In addition, many kidney diseases present a broad range of morphological alterations, which in sum reflect the morphologic and pathophysiologic complexity of glomerulopathies [10] (Sup. Figure 1). Maybe the most extreme example for this diagnostic complexity is the classification of lupus nephritis into different classes, each exhibiting a characteristic, however not pathognomonic, morphological signature [10-13]. Inline, other diagnostic entities may demonstrate a substantial variation in their morphology patterns that frequently reflect their clinical behavior (for instance, $\operatorname{Ig}$ A nephropathy may also show an endocapillary in addition to the mesangial hypercellularity and necrosis at the same time). In this study, CNN models were trained to classify different glomerular lesions in a way that imitates the education of pathology residents to discern disease patterns before making the diagnosis [10-12]. Our approach was based on the digitalization of slides from the routine diagnostics and isolation of glomeruli. We demonstrate that it can successfully identify the glomerular lesions and recognize more than one disease pattern when confronted with complex glomerular changes.

\section{Methods}

\section{Patient specimen and raw data generation}

Periodic acid-Schiff (PAS) stained formalin-fixed paraffinembedded tissue was retrieved (Institute of Pathology, Medical Faculty Mannheim, Heidelberg University \& Institute of Pathology, University Medical Center of the Johannes Gutenberg University Mainz) and used in an anonymized way. The data collection and all experiments were conducted in accordance with a vote of the ethics commission II of the Heidelberg University (vote 2020-847R).

\section{Data management and analysis}

Image processing (glomerulus segmentation, image cropping) and preprocessing were performed (image data arrangement) in the MATLAB environment (MATLAB (R2017a). Machine learning was performed in Python with PyTorch 14, 15 as described in the corresponding sections.

\section{Defining the patterns of glomerular changes}

We first categorized the basal morphologic patterns of glomerular alterations for our $\mathrm{CNN}$-model-based approach: normal glomerulus (pattern 01), amyloidosis (pattern 02), nodular sclerosis (pattern 03), global sclerosis (pattern 04), mesangial hypercellularity (pattern 05), mesangioproliferative glomerulonephritis (MPGN) (pattern 06), necrosis/ crescent (pattern 07), and segmental sclerosis (pattern 08) (Fig. 1 and Sup. Figure 1). Extraglomerular structures that should offer an "exit strategy" for the models were labeled as pattern 09 (Fig. 1 and Sup. Figure 1-2). The categories represent prototypical glomerular changes in terms of light microscopy and the experiments do not rely on any ancillary studies that would have been implemented in routine diagnostics (such as immunohistochemical, immunofluorescence, or ultrastructural investigations). For example the typical pattern "amyloidosis" with its acellular, weakly PAS-positive depositions needs further investigations such as Congo-red stain (or EM) to establish the diagnosis and to exclude its mimics (such as fibrillary glomerulopathy).

\section{Creation of three datasets for training, validation, and testing}

We digitalized PAS stained slides of kidney biopsies from routine diagnostics from two independent institutions (Institute of Pathology Mannheim and Institute of Pathology Mainz). In total, three datasets (datasets \#1 and \#2 for training and validation and dataset \#3 for testing only) were defined (Sup. Figure 2): 
Fig. 1 Paradigmatic patterns of glomerular diseases. In terms of conventional morphology, the fundamental glomerular changes were attributed to 9 patterns (Nrs. 01-09). Extraglomerular structures were labeled as 'default' pattern 09. MPGN, membranoproliferative glomerulonephritis
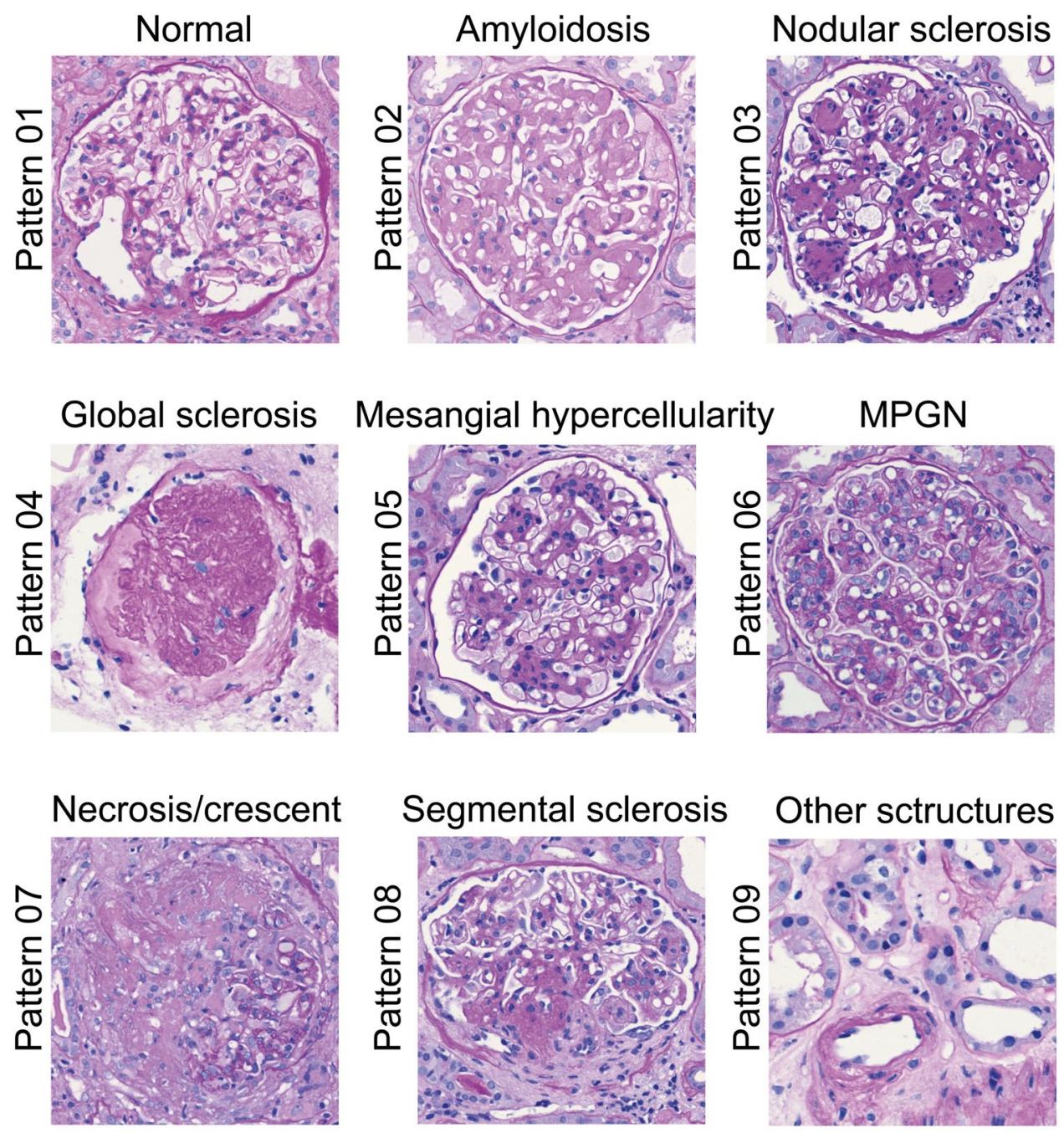

Dataset \#1: To foster the collection of glomeruli from the above-mentioned scanned cases, we implemented a glomerulus detection RCNN-based object detection tool (as published by Kawazone et al. [16]). This tool automatically cropped all glomeruli per whole slide image. In the next step, an expert nephropathologist (SP) assigned a total of 12,253 images of cropped glomeruli with a single morphological pattern to the predefined patterns (Fig. 2) sorting out incomplete glomeruli or images with tissue artifacts. The numbers per pattern range from 70 for pattern 06 (MPGN) to 4385 for pattern 01 (normal glomerulus) (Sup. Figure 4 A1-2).

Datasets \#2-3: A total of 11,142 newly cropped glomeruli were assigned to the predefined patterns in the consensus of three independent nephropathologists (SP, ZVP, MMG). The numbers per pattern range from 46 for pattern 06 (MPGN) to 4091 for pattern 01 (normal glomerulus) (Sup. Figure 4 B1-2). From this database, 20 images per pattern were chosen as the test set (hereafter dataset \#3) and kept away until testing the models (Sup. Figure 4C). The remaining images (hereafter dataset \#2) were used to re-train and validate the models pre-trained on dataset \#1. All three nephropathologists performed their categorization of the images prior to the learning and testing phase, thus, not being influenced by any results of the $\mathrm{CNN}$ algorithm.

\section{CNN-training}

Several published classification models or versions of published models (AlexNet [17], VGG [18], ResNet [19], Densenet [20], Squeeze net [21], and Inception net [22]) were trained (Sup. Figure 3). By using the repository hiddenlayer the architecture of the herein applied models can be visualized (as done in the folder ModelPlots in the according GitHub-repository cited below).'

The image size was set to $224 \times 224$ pixel for all models except the Inception net, where it was set to $299 \times 299$ pixel. As optimizer we used a stochastic gradient descent [23, 24]. The initial learning rate was $10^{-3}$. We used a learning rate 

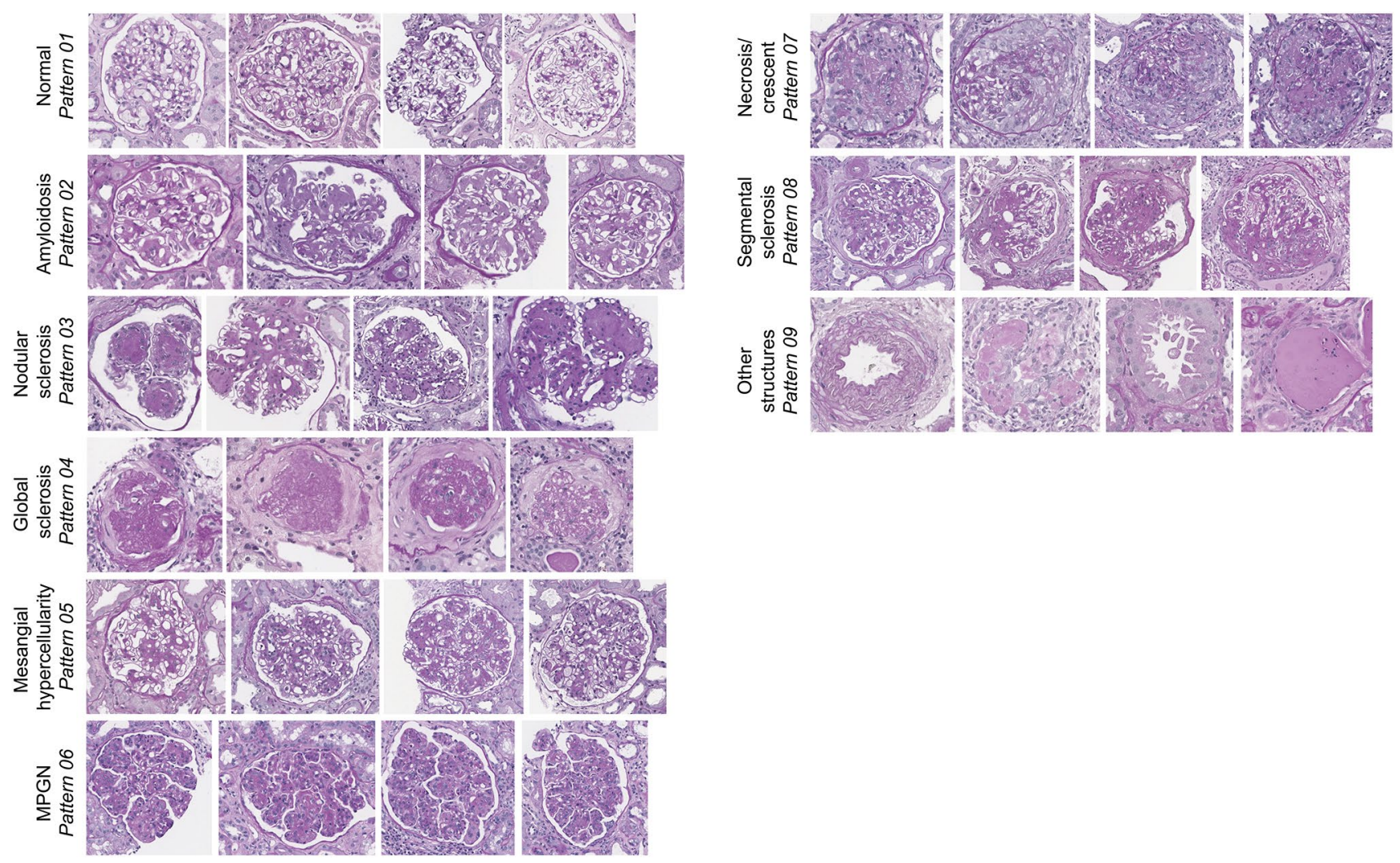

Fig. 2 Examples of processed images. For each pattern, four examples from the image database are shown. The different image proportions are given by different bounding boxes reflecting the variable glomerular shape and size in the sections

decay of 0.1 every 7 epochs. All models were trained for 50 epochs. The loss function was cross entropy loss.

All calculations were performed on a GPU (Graphics Processing Unit) (NVidia TitanXP).

In a typical transfer learning setting a complex, published model, which initially was trained on a large dataset (e.g. the cityscape dataset), is re-trained on a smaller dataset. The idea behind this is that complex models may not converge if trained only on a small dataset. By using a pre-trained model, this model has already learned many features and usually only needs to re-learn how to map the features on the new classes. Therefore, in a typical transfer learning setting only the last layers of a CNN model are re-trained [25-27]. However, in our model, re-training of the last layer solely did not lead to a convergence of the loss, likely due to a specific image composition of the kidney tissue [28-30] in contrast to large image datasets usually used for training. For instance, histological images have quite a different color composition in contrast to cityscapes.

\section{Statistical analysis}

Statistical analysis was performed in R version 3.2.4 [31]. Kappa coefficients were calculated to assess the degree of agreement for binary factors. In the case of more than two raters, the Fleiss' kappa was calculated. As a measure for classification quality, the accuracy and the confusion matrix are presented.

\section{Code and data availability}

The according code is available on GitHub: https://github. com/catweis/Assessment-of-glomerular-morphologicalpatterns-by-deep-learning.

The trained models and the test dataset are available on HeiData: https://heidata.uni-heidelberg.de/privateurl.xhtml? token $=6 f 166 c a 5-c 48 a-4943-86 c 7-4 b 35 c 88 d c 879$.

\section{Results}

\section{Training and validation of different convolutional neuronal network (CNN) classification models}

We used different published classification models [14, 15] and the aforementioned dataset \#1 (defined by one expert pathologist) and dataset \#2 (consensus of three expert pathologists) to classify glomerular disease patterns. In 
addition, we aimed to compare the performance of different convolutional neuronal networks CNNs in terms of accuracy. To this end, several versions of AlexNet [17], VGG [18], ResNet [19], Densenet [20], Squeeze net [21], and Inception net [22] were trained (Sup. Figure 2-3). Examples of the model input are shown in Fig. 2.

The classification quality for all networks was assessed based on the untrained part (20\%) of datasets \#1 and \#2 used for validation purposes (hereinafter validation sets \#1 and \#2). No cross-validation approach was used; thus, the images within the validation sets are unseen. The accuracy and kappa value were calculated based on the resulting confusion matrices. The accuracy values ranged from 0.910 to 0.920 (validation set \#1) and respectively 0.944-0.984 (validation set \#2). The kappa values ranged from 0.884 to 0.933 (validation set \#1) and respectively 0.927-0.979 (validation set \#2). In summary, the ResNetvariant ResNet101 produced the best results for the validation sets (Table 1 and Fig. 2).

In order to measure the classification accuracy for each pattern separately, Fleiss' kappa-value was calculated in all trained models. Independently of the model used, the best accordance (i.e., highest kappa-values) could be observed for the normal glomerulus (pattern 01;0.967), MPGN (pattern 06; 0.985), and other structures (pattern 09; 0.0971). In contrast, the values were lowest for mesangial hypercellularity (pattern 05; 0.863). All remaining kappa-values were in the range in between.

Table $1 \mathrm{CNN}$-model results of the validation dataset (each part of dataset \#1 and \#2)

\begin{tabular}{lll}
\hline Model & Accuracy & Kappa value \\
\hline AlexNet [17] & $0.910 / 0.944$ & $0.884 / 0.927$ \\
squeeznet [21] & $0.912 / 0.945$ & $0.886 / 0.928$ \\
vgg11 [18] & $0.932 / 0.963$ & $0.912 / 0.951$ \\
vgg19 [18] & $0.940 / 0.968$ & $0.912 / 0.958$ \\
ResNet18 [19] & $0.940 / 0.970$ & $0.922 / 0.960$ \\
vgg16 [18] & $0.933 / 0.970$ & $0.913 / 0.960$ \\
ResNet34 [19] & $0.954 / 0.975$ & $0.940 / 0.967$ \\
ResNet50 [19] & $0.949 / 0.977$ & $0.934 / 0.970$ \\
inception [22] & $0.947 / 0.980$ & $0.930 / 0.973$ \\
densenet121 [20] & $0.955 / 0.980$ & $0.941 / 0.974$ \\
ResNet152 [19] & $0.947 / 0.981$ & $0.932 / 0.975$ \\
ResNet101 [19] & $0.949 / 0.984$ & $0.933 / 0.979$ \\
\hline
\end{tabular}

From dataset \#1 ( $\mathrm{n}=2451$ images $)$ and from dataset \#2 $(n=2267)$, each corresponding to $20 \%$, are used for validation only. The table below shows the accuracy and kappa-values for different models, with the first value for dataset \#1 and the second for dataset \#2

\section{Testing of the trained CNN-models}

The test dataset-dataset \#3-contained 180 images assigned to the predefined patterns by consensus of three independent expert nephropathologists (MMG, SP, and ZVP). Of note, in contrast to the previously used validation sets, the test set was balanced with 20 images per pattern. The images were analyzed using all the models mentioned above, and the accuracy and kappa value were calculated based on the resulting confusion matrices. The accuracy values ranged from 0.856 to 0.944 , and the kappa values ranged from 0.838 to 0.938 . In summary, the ResNet-variant ResNet152 (Fig. 3) produced the best results for the test set (Table 2). The Fleiss' kappa values reached the best accordance for MPGN (pattern 06; 0.985) and the worst for mesangial hypercellularity (pattern 05; 0.756).

\section{Identification of the image areas decisive for the CNN-decision making}

In order to elucidate the CNN-based classification process, we strived to identify which aspects of the image are essential for decision making. We implemented two different approaches:

1. First, we used a class activation map (CAM) to produce a heat map for the network's highest activation, leading to the correct class assignment for every image [25]. We applied a free-available Pytorch-implementation from GitHub [26]. At one end of the spectrum, in the setting of normal glomerulus (pattern 01), the model

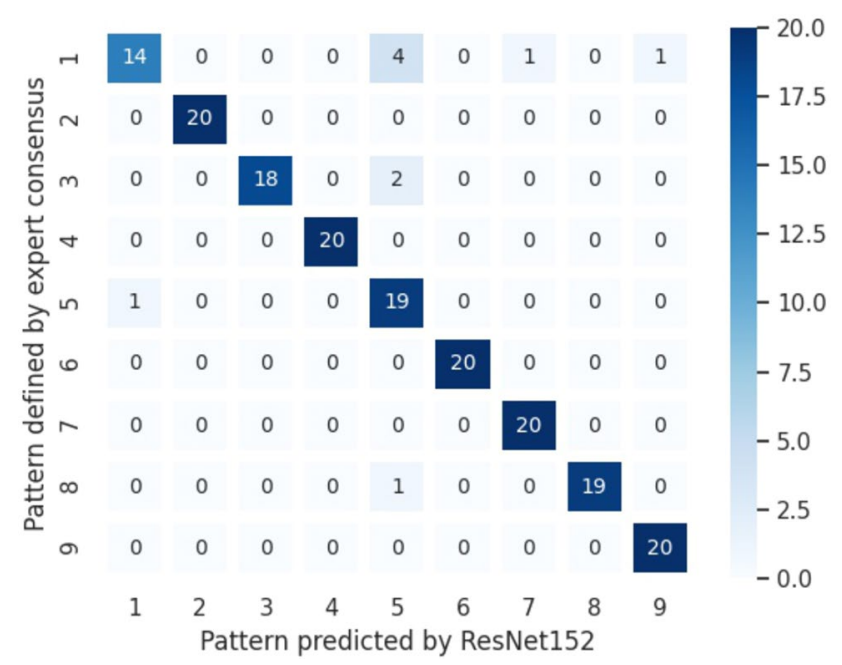

Fig. 3 Performance of the CNN algorithm. Confusion matrix depicting the results of $\mathrm{CNN}$-based glomerular categorization (by the ResNet 152) on the x-axis compared with the expert consensus on the $y$-axis. The herein analyzed test set contains 20 images per pattern. Here the accuracy was 0.944 , and the kappa-value 0.938 
Table 2 Results for the test dataset (dataset \#3)

\begin{tabular}{lll}
\hline Model & Accuracy & Kappa value \\
\hline AlexNet [17] & 0.856 & 0.838 \\
squeeznet [21] & 0.861 & 0.844 \\
ResNet50 [19] & 0.900 & 0.888 \\
ResNet101 [19] & 0.900 & 0.888 \\
$\operatorname{vgg} 11[18]$ & 0.911 & 0.900 \\
$\operatorname{vgg} 19[18]$ & 0.911 & 0.900 \\
ResNet18 [19] & 0.917 & 0.906 \\
ResNet34 [19] & 0.928 & 0.919 \\
densenet121 [20] & 0.928 & 0.919 \\
inception [22] & 0.928 & 0.919 \\
vgg16 [18] & 0.939 & 0.931 \\
ResNet152 [19] & 0.944 & 0.938 \\
\hline
\end{tabular}

Dataset \#3 comprises $n=180$ images that were categorized by three nephropathologists (MMG, SP, and ZVP). For the trained models, the accuracy and the kappa value were calculated mainly focused on the edges of the image, where small parts of the tubulointerstitium and the blood vessels are shown (Fig. 4A), implying that recognition of normal glomerulus may be an indirect result of the exclusion of non-glomerular structures and incomplete glomeruli. On the other hand, in the case of amyloidosis (pattern 02), the heat maps showed that the model directly screened amyloid deposition within the glomerulus and, interestingly, in the interstitial blood vessels (in Fig. 4B). Also, in other patterns, the heat maps apparently directly highlighted the pathological regions within the image.

2. Second, we used a deep convolutional generative adversarial network (DCGAN) to produce example images for each class. The code was derived from the book by Hany and Walters [27] and is publicly available on GitHub [32]. The model consists of two networks: a generator learns to create synthetic image samples from a given distribution, and a discriminator being a classifier that distinguishes between authentic images from the given dataset and the fake ones created by the generator. Both
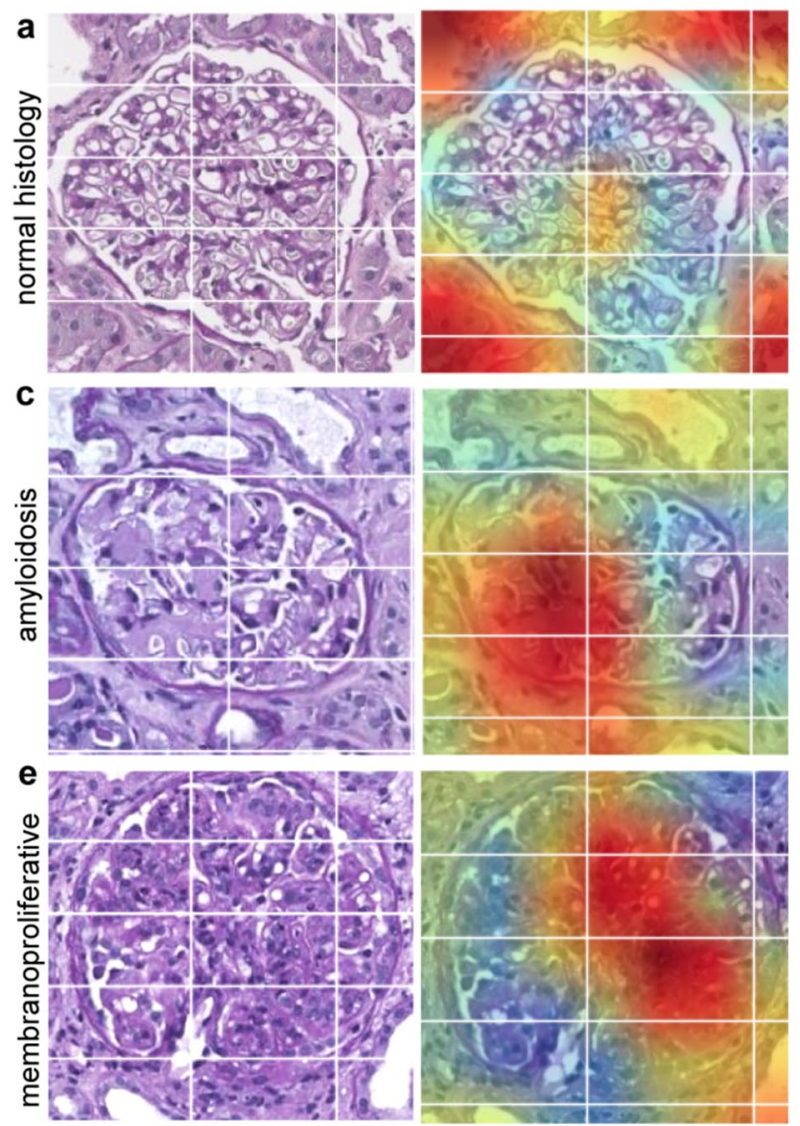

Fig. 4 Class activation maps (CAM) visualizing the decision-relevant image parts. In the trained $\mathrm{CNN}$ model, CAM was used to detect the image regions responsible for the strongest activation of the corresponding class. Shown are selected glomerular disease patterns along with a heat map visualizing areas in dark red that were most decisive
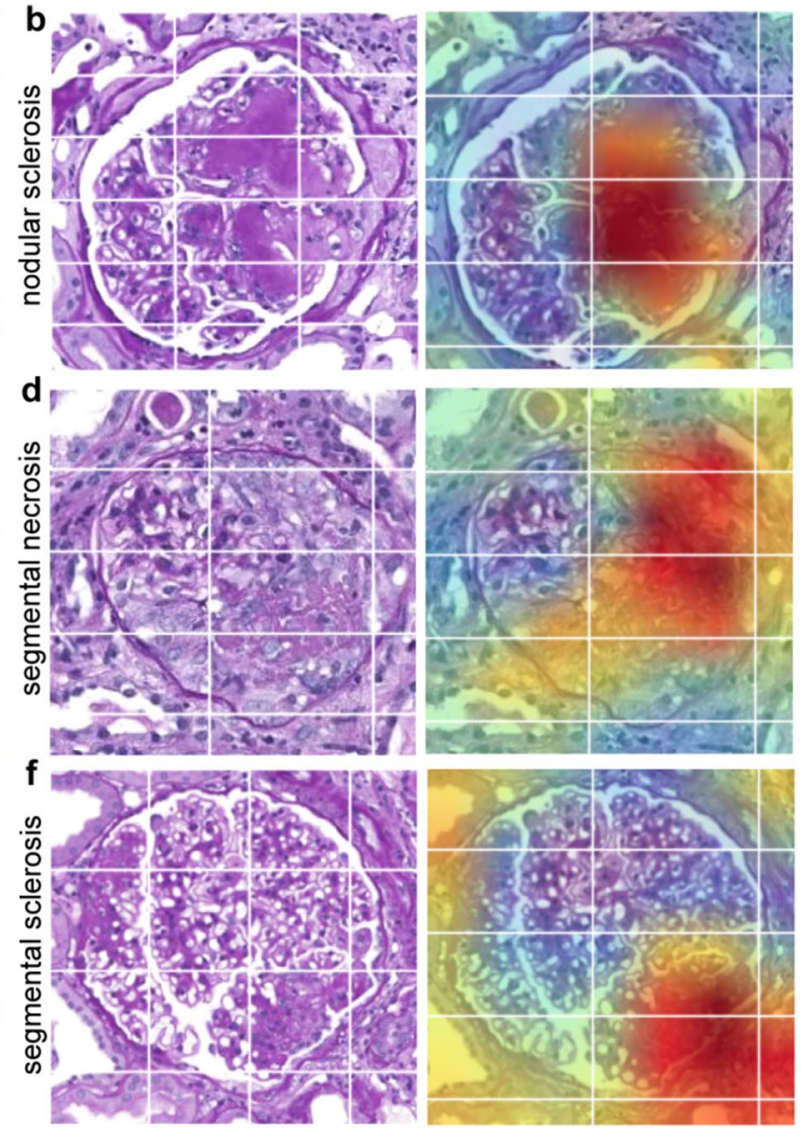

for the class activation. In $\mathbf{b}-\mathbf{f}$, these areas are congruent with the foci of morphological alteration. In panel a (normal glomerulus), there was no pathology, and therefore no activation of the model trained on pathology classification 
networks are trained simultaneously in an adversarial process in which the generator tries to deceive the discriminator while the discriminator tries to identify the fake images. For normal glomeruli (pattern 01), this approach led to blurred but rather specific images, in the sense that the glomeruli were recognized as more or less physiological (Supp. Figure 5A). The images were also rather specific for global sclerosis (pattern 04) (Supp. Figure 5D). However, in both cases, the herebyproduced images were easily recognized as non-original due to their limited image quality. For the other patterns, the produced images were non-distinguishable. The network generated blurred images for all analyzed classes (Supp. Figure 5).

\section{Application of the trained model in diagnostic settings}

In practical terms, a kidney biopsy can contain glomeruli that do not necessarily show the same disease pattern and are heterogeneously distributed; for example, a renal biopsy from a patient with nephrosclerosis may contain normal, segmentally, and globally sclerosed glomeruli. Similarly, a case of necrotizing IgA glomerulonephritis may show mesangial expansion, endocapillary proliferation, necrosis, as well as segmentally and globally sclerosed glomeruli.

Thus, to determine how the algorithm will deal with real biopsy cases, we manually cropped glomeruli from 46 diagnostic slides and assigned them to one of the previously defined disease patterns.

The trained ResNet152-model evaluated every cropped image for all cases and produced a binary output: the predicted value can be within (correct choice) or outside the expected range (false choice). The mean correct rate was 0.867 with a standard deviation of 0.169 . Pattern 09 (other structures) as default pattern was among the wrong choices, the most frequent one. Pattern distributions per diagnosis and diagnosis group were plotted per case in a heat map (Supp. Figure 6). Some categories with a very characteristic morphological signature (like amyloidosis) tended to be assigned correctly, whereas other morphologically more similar patterns such as mesangial hypercellularity and nodular sclerosis showed poorer classification quality.

\section{Detection of more than one disease pattern in a single glomerulus}

A synchronous combination of different morphological patterns can occur both on the whole biopsy and on the single glomerulus level, for example, segmental necrosis in MPGN or segmental sclerosis in a glomerulus with mesangial hypercellularity. To test the model's decision on such glomeruli with more than one pattern, we used new images of glomeruli with complex changes that cannot be attributed to any single category but show facets of several patterns in parallel. For every input image, the model produced a probability value for all nine classes ranging from 0 to 1 . As shown in Fig. 5, the algorithm "recognizes" more than one disease pattern reflected by approximately equally high probabilities of the relevant patterns. Thus, in a case of IgA-glomerulonephritis with endocapillary hypercellularity and incipient segmental sclerosis (Fig. 5A), the 'mesangial hypercellularity' and 'segmental sclerosis' were identified along with a similarity to the 'MPGN' pattern that reflects the endocapillary hypercellularity in this case. Of note, here and in most of the analyzed ambivalent cases, the basketcategory 'other structures' received a relatively high ratingnot surprising if appreciating that the training was performed on 'single patterned' images. In the next step, we selected a more complex image from a case of IgA-nephropathy showing a combination of mesangial and extracapillary proliferation progressing to sclerosis (Fig. 5B). Our model correctly reflected the fact that the sclerosis reached the border between segmental and global (i.e. 50\% of the glomerular area) by assigning approximately equal ratings for the patterns of segmental and global sclerosis. Indeed, as collapsed capillaries with extracapillary proliferation dominate here, discrete mesangial hypercellularity in this case was not recognized by the network. Interestingly, the homogeneous fibrous tissue "resembled" amyloidosis to some extent (Fig. 5B). In a third case of a MPGN with a small necrosis progressing to segmental sclerosis, we could observe accurate categorization of all the relevant, even discrete patterns (i.e. 'MPGN', 'mesangial hypercellularity, 'necrosis/crescent' and 'segmental sclerosis').

\section{Discussion}

In the present study, we investigated the potential value of machine learning in automatized recognition of common glomerular morphological changes. As quality parameter for the classification, we used the well-established kappa-value, a classical tool to measure inter-rater reliability and agreement for nominal (diagnostic) decisions by (human) experts. Importantly, we initially intended to exclude the influence of interobserver variability (as one of the hallmarks of nephropathology) and focus on the capacity of CNN to recognize (even complex, but) clear-cut patterns. Hence, our models were first trained on a dataset initially defined by one expert pathologist, in the next phase, they were re-trained on a second dataset defined based on a three-expert consensus and finally evaluated on a subpart of the second, three-pathologist-consensus dataset (Sup. Figure 2). Whereas calculation of the accuracy in our study provided an overall measurement for the classification quality, kappa statistics mirrored 
A1

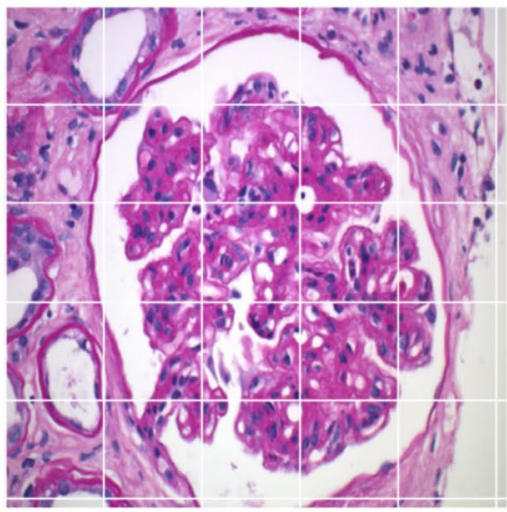

B1

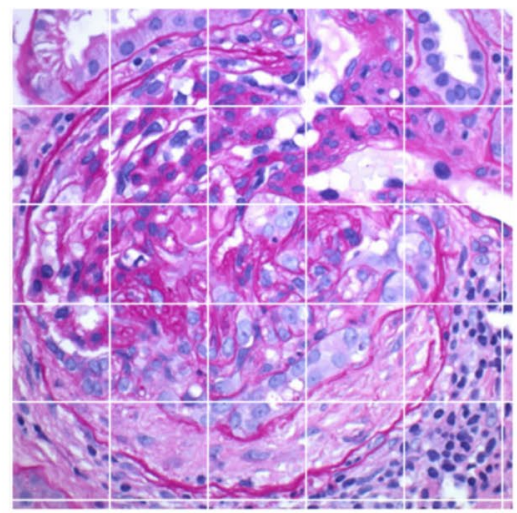

C1

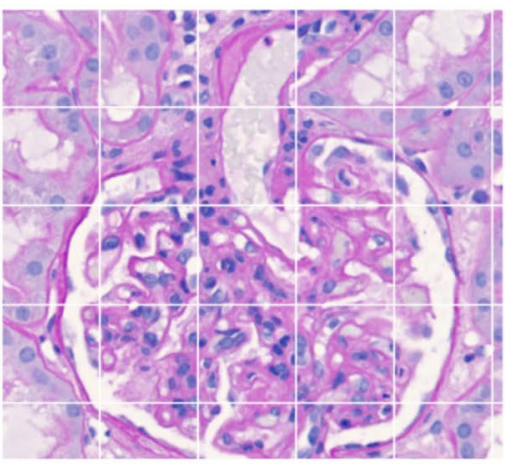

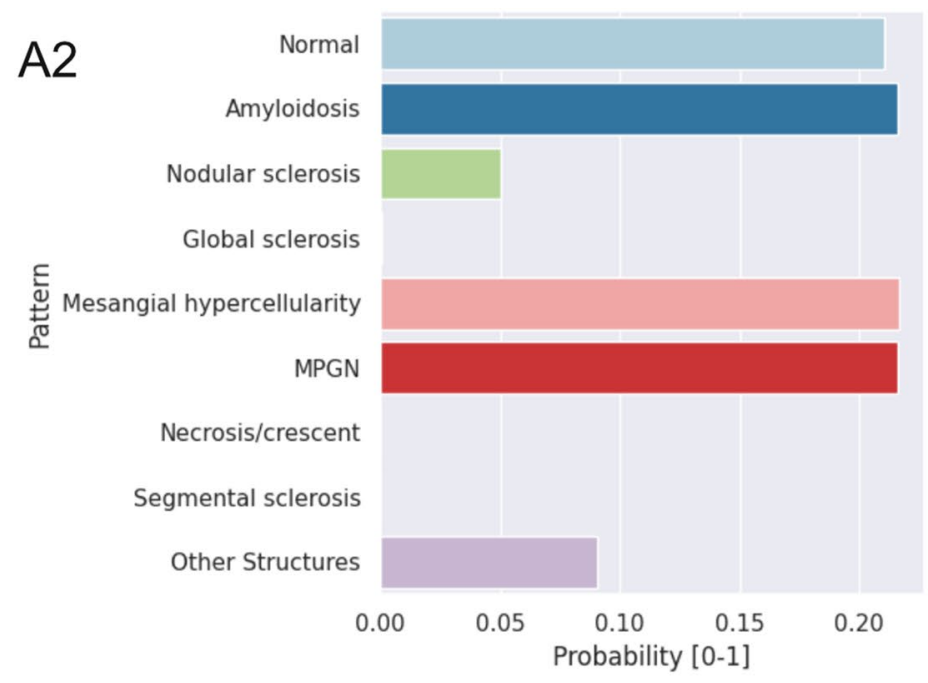

B2

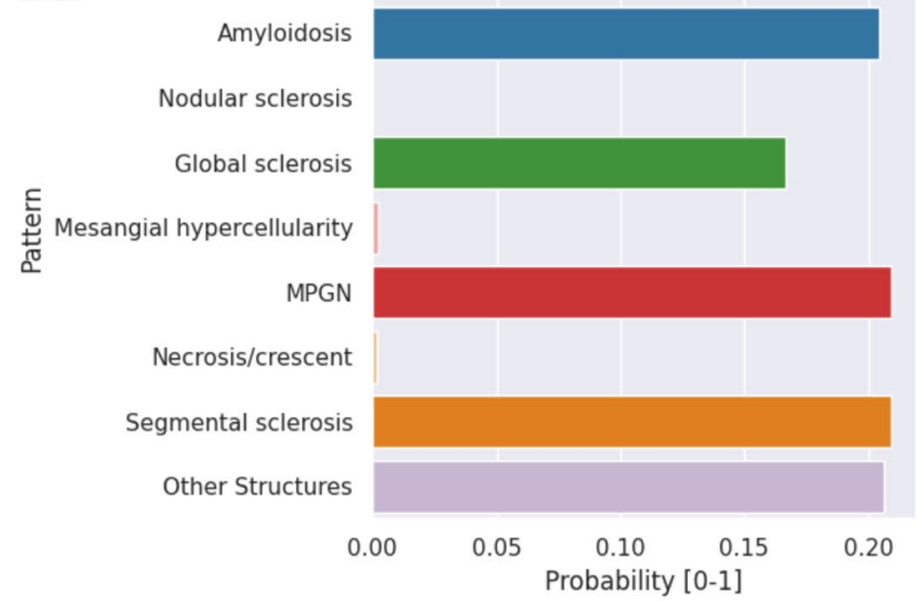

C2

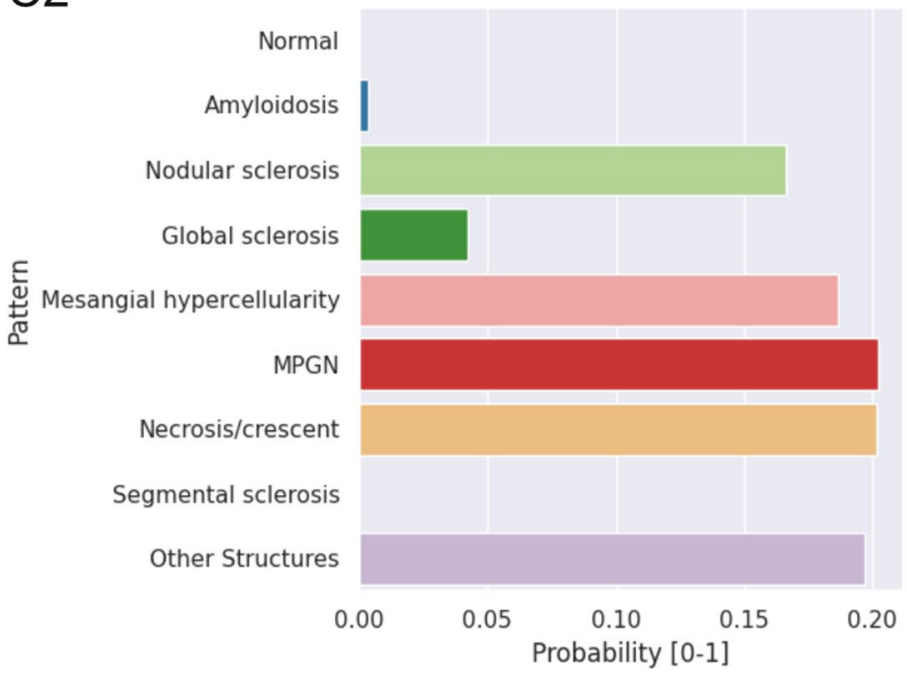

kappa-values from 0.884 (AlexNet-model) $[14,15,17]$ to 0.979 (ResNet 101-model) [14, 15, 19] for the validation datasets, hence demonstrating good to very good accordance 
4Fig. $5 \mathrm{CNN}$-based algorithms are capable of recognizing several coincident disease patterns in one glomerulus in "real-life" images. Images of glomeruli with more than one disease pattern were taken from the daily diagnostic routine and subjected to $\mathrm{CNN}$ analysis. This generated probability values ranging from 0 to 1 , reflecting the 'similarity' to the nine classes for each image. A IgA-glomerulonephritis with endocapillary hypercellularity and incipient segmental sclerosis. B IgA-glomerulonephritis with extracapillary proliferation progressing to sclerosis that reaches the border between segmental and global. C MPGN with small extracapillary proliferation progressing to segmental sclerosis

with the provided expert opinion. Furthermore, in a dedicated test set with images evaluated and selected by the consensus of three experts from two independent institutes, the kappa-values ranged as well from good to very good $(0.838$ for the AlexNet-model [14, 15, 17] to 0.938 for the ResNet 152-model [14, 15, 19]).

As expected, successful recognition of morphological patterns was not sufficient for an accurate assignment to the appropriate diagnosis in most cases (Sup. Figure 1). A typical example of a glomerular disease with a limited set of pathological changes is amyloidosis (pattern 02). Although the establishment of the diagnosis "amyloidosis" and the exclusion of its mimics (e.g. the rare fibrillary glomerulopathy) need further investigations (such as Congo-red-stain or electron microscopy), the amyloidosis-pattern is quite characteristic. Accordingly, the diagnosis of amyloidosis was recognized by the ResNet152 in the diagnostic test set with $100 \%$ accuracy. On the other hand, immune-complex glomerulonephritides like IgA glomerulonephritis, lupus nephritis with a spectrum of rather unspecific lesion patterns obviously require further input from immunohistochemical/ immunofluorescence and ultrastructural studies as well as clinical data for the completion of the diagnostic process.

The comparison of the performance of our trained models with other classification models is hampered by several points: many popular image datasets (like CIFAR-10) do not correspond to our model regarding the image content since they contain roughly defined morphological classes ('dog', 'sheep', etc.) that are not comparable with rather delicate alterations of glomerular histology. Next, the data pool in our study comprised in total 23,395 images assigned to nine classes. As a comparison, broadly used datasets like the ImageNet 2012 classification dataset cover more than 1000 classes in more than 1 million images [33]. Furthermore, even if certain published datasets contain histological images (for instance, the Atlas of Digital Pathology dataset with a significantly larger image cluster-17,668 images grouped into 42 classes), the image content and the task definition (recognition of the histological tissue type) substantially differ from our model [34]. We focused on highly specific glomerular architecture and set the task to recognize proportionally discrete pathological changes.
Moreover, the comparison of our results to other published models is hampered by the differences regarding the underlying data and partially by the metrics employed for the classification quality. As generally accepted, we applied accuracy as an overall measure for the classification quality. Considering the above-mentioned work by Hosseini et al. [34] that reported an accuracy $\geq 0.95$ for all used models, our results from a proportionally much smaller dataset (accuracy values $0.856-0.944$ for the test set) are comparable. Nevertheless, dealing with a diagnostic problem, we also applied typical inter-rater statistical values like the kappavalue and Fleiss kappa-value, used only in a minority of papers. For instance, in a trained CNN-model for the classification of diabetic retinopathy in fundus images, Gyatri et al. [35] reported a kappa-value for multiclass classification of 0.994. The kappa-values for the multiclass problem of the nine patterns in our study were in the range $0.756-0.985$. In summary, the herein tested models can distinguish between most of the patterns with very high accuracy.

Importantly, our models were first trained on a dataset initially defined by one expert pathologist, and in the next phase they were re-trained on a second dataset defined based on a three-expert consensus. Finally, the models were evaluated on a test set that is a subpart of the second, threepathologist-consensus dataset. There, the kappa-values for all herein trained and tested models are in the range from good to very good.

The major challenge of glomerular pathology is that a single glomerulus can bear a combination of different disease patterns. This particular problem of overlapping tasks distinguishes our project from other approaches that trained CNNs to categorize tumors in mutually exclusive, rigorous categories like tumor type, microsatellite stability, or prognostic category [36, 37]. Here we could demonstrate that our CNN model dealt with complex cases derived from routine diagnostics effectively, and recognized several co-existing lesion patterns occurring in a single glomerulus.

It is important to stress that-similar to routine diagnostics-the recognition of a particular glomerular pattern does not equal the diagnosis which is the result of a complex process of considering ancillary tissue studies, clinical information, serological data and patient's history. For example, this input is essential for the interpretation of mesangial hypercellularity that can be the manifestation of an IgAglomerulonephritis, lupus nephritis or another connectivetissue disease. Similarly, the light-microscopic pattern "normal glomerulus" might still lead to the diagnosis of an early membranous glomerulonephritis or minimal-change glomerulopathy if the characteristic ultrastructural findings and proteinuria are present.

In summary, our model shows that artificial intelligence may be used as a reliable model in the up-front evaluation of kidney pathology in biopsy specimens. Moreover, the 
presented algorithm is capable of "recognizing" the affection of a single glomerulus by more than one disease patterna situation regularly encountered in diagnostic nephropathology. Further studies are warranted to integrate also the immunohistochemical and ultrastructural patterns together with clinical parameters (such as proteinuria, erythrocyturia, kidney function, and serum parameters) to boost the accuracy of digital learning in nephropathology and to present a valuable tool applicable in routine diagnostics in the future.

Supplementary Information The online version contains supplementary material available at https://doi.org/10.1007/s40620-021-01221-9.

Acknowledgements The authors also thank the IT department staff of Medical Faculty Mannheim and especially Mr. Bohne-Lang for supervising the computer administration and infrastructure. The authors gratefully acknowledge the data storage service SDS@hd supported by the Ministry of Science, Research and the Arts Baden-Württemberg (MWK) and the German Research Foundation (DFG) through grant INST 35/1314-1 FUGG and INST 35/1503-1 FUGG.

Author contributions C-AW: study design, data formation, model training, statistical evaluation, manuscript drafting. JNB and JV: data preparation and model training. MR: model training and statistical evaluation. SH: statistical evaluation and manuscript drafting. MG: data generation and manuscript drafting. ZVP and SP: study design, data generation, statistical evaluation, manuscript drafting.

Funding Open Access funding enabled and organized by Projekt DEAL.

\section{Declarations}

Conflict of interest All authors declare that they have no conflict of interest. This project did not receive any specific funding.

Ethical approval The data collection and all experiments were conducted in accordance with a vote of the ethics commission II of the Heidelberg University (vote 2020-847R).

Informed consent For this type of study, formal consent is not required.

Open Access This article is licensed under a Creative Commons Attribution 4.0 International License, which permits use, sharing, adaptation, distribution and reproduction in any medium or format, as long as you give appropriate credit to the original author(s) and the source, provide a link to the Creative Commons licence, and indicate if changes were made. The images or other third party material in this article are included in the article's Creative Commons licence, unless indicated otherwise in a credit line to the material. If material is not included in the article's Creative Commons licence and your intended use is not permitted by statutory regulation or exceeds the permitted use, you will need to obtain permission directly from the copyright holder. To view a copy of this licence, visit http://creativecommons.org/licenses/by/4.0/.

\section{References}

1. Uzunova H, Schultz S, Handels H, Ehrhardt J (2019) Unsupervised pathology detection in medical images using conditional variational autoencoders. Int J Comput Assist Radiol Surg 14:451-461. https://doi.org/10.1007/s11548-018-1898-0

2. Becker JU, Mayerich D, Padmanabhan M, Barratt J, Ernst A, Boor P, Cicalese PA, Mohan C, Nguyen HV, Roysam B (2020) Artificial intelligence and machine learning in nephropathology. Kidney Int 98:65-75. https://doi.org/10.1016/j.kint.2020.02.027

3. Kriegsmann M, Kriegsmann K, Steinbuss G, Zgorzelski C, Kraft A, Gaida MM (2021) Deep learning in pancreatic tissue: identification of anatomical structures, pancreatic intraepithelial neoplasia, and ductal adenocarcinoma. Int J Mol Sci 22:5385

4. Hermsen M, de Bel T, den Boer M, Steenbergen EJ, Kers J, Florquin S, Roelofs J, Stegall MD, Alexander MP, Smith BH et al (2019) Deep learning-based histopathologic assessment of kidney tissue. J Am Soc Nephrol 30:1968-1979. https://doi.org/10.1681/ ASN.2019020144

5. Bukowy JD, Dayton A, Cloutier D, Manis AD, Staruschenko A, Lombard JH, Solberg Woods LC, Beard DA, Cowley AW Jr (2018) Region-based convolutional neural nets for localization of glomeruli in trichrome-stained whole kidney sections. J Am Soc Nephrol 29:2081-2088. https://doi.org/10.1681/ASN.2017111210

6. Ginley B, Lutnick B, Jen KY, Fogo AB, Jain S, Rosenberg A, Walavalkar V, Wilding G, Tomaszewski JE, Yacoub R et al (2019) Computational segmentation and classification of diabetic glomerulosclerosis. J Am Soc Nephrol 30:1953-1967. https://doi. org/10.1681/ASN.2018121259

7. Zeng C, Nan Y, Xu F, Lei Q, Li F, Chen T, Liang S, Hou X, Lv $\mathrm{B}$, Liang D et al (2020) Identification of glomerular lesions and intrinsic glomerular cell types in kidney diseases via deep learning. J Pathol 252:53-64. https://doi.org/10.1002/path.5491

8. Chen Y, Zee J, Smith A, Jayapandian C, Hodgin J, Howell D, Palmer M, Thomas D, Cassol C, Farris AB 3rd et al (2021) Assessment of a computerized quantitative quality control tool for whole slide images of kidney biopsies. J Pathol 253:268-278. https://doi.org/10.1002/path.5590

9. Uchino E, Suzuki K, Sato N, Kojima R, Tamada Y, Hiragi S, Yokoi H, Yugami N, Minamiguchi S, Haga H et al (2020) Classification of glomerular pathological findings using deep learning and nephrologist-AI collective intelligence approach. Int J Med Inform 141:104231. https://doi.org/10.1016/j.ijmedinf.2020.104231

10. Fogo A, Bruijn JA, Cohen AH, Colvin RB, Jennette JC (2007) Fundamentals of renal pathology. Springer, New York

11. Colvin RB, Chang A (2019) Diagnostic pathology: kidney diseases. Elsevier, Berlin

12. Goebel H, Wiech T (2012) Diagnostik von Erkrankungen der Eigenniere-für Einsteiger. In: Deutsche Division der IAP

13. Tang Y, Zhang W, Zhu M, Zheng L, Xie L, Yao Z, Zhang H, Cao D, Lu B (2018) Lupus nephritis pathology prediction with clinical indices. Sci Rep 8:10231. https://doi.org/10.1038/ s41598-018-28611-7

14. Paszke A, Gross S, Chintala S, Chanan G, Yang E, DeVito Z, Lin Z, Desmaison A, Antiga L, Lerer A (2017) Automatic differentiation in pytorch

15. Paszke A, Gross S, Massa F, Lerer A, Bradbury J, Chanan G, Killeen T, Lin Z, Gimelshein N, Antiga L (2019) Pytorch: An imperative style, high-performance deep learning library, $\mathrm{pp}$ 8026-8037

16. Kawazoe Y, Shimamoto K, Yamaguchi R, Shintani-Domoto Y, Uozaki H, Fukayama M, Ohe K (2018) Faster R-CNN-based glomerular detection in multistained human whole slide images. $\mathrm{J}$ Imaging 4:91 
17. Krizhevsky A (2014) One weird trick for parallelizing convolutional neural networks. arXiv:1404.5997

18. Simonyan K, Zisserman A (2014) Very deep convolutional networks for large-scale image recognition. arXiv: 1409.1556

19. He K, Zhang X, Ren S, Sun J (2020) Deep residual learning for image recognition, pp 770-778

20. Huang G, Liu Z, Van Der Maaten L, Weinberger KQ (2017) Densely connected convolutional networks, pp 4700-4708

21. Iandola FN, Han S, Moskewicz MW, Ashraf K, Dally WJ, Keutzer K (2016) SqueezeNet: AlexNet-level accuracy with 50x fewer parameters and $<0.5 \mathrm{MB}$ model size. arXiv: 1602.07360

22. Szegedy C, Vanhoucke V, Ioffe S, Shlens J, Wojna Z (2016) Rethinking the inception architecture for computer vision, pp 2818-2826

23. SGD-PyTorch 1.9.1 documentation. https://pytorch.org/docs/ stable/generated/torch.optim.SGD.html\#torch.optim.SGD

24. Sutskever I, Martens J, Dahl G, Hinton G (2020) On the importance of initialization and momentum in deep learning, $p 14$

25. Zhou B, Khosla A, Lapedriza A, Oliva A, Torralba A (2016) Learning deep features for discriminative localization, 27-30 June 2016, pp 2921-2929

26. Schniertshauer J (2018) pytorch-CAM. https://github.com/joe31 41592/PyTorch-CAM

27. Hany J, Walters G (2019) Hands-on generative adversarial networks with PyTorch 1. In: Implement next-generation neural networks to build powerful GAN models using Python. Packt Publishing Ltd

28. Koehrsen W (2018) Transfer learning with convolutional neural networks in PyTorch: how to use a pre-trained convolutional neural network for object recognition with PyTorch. https://towar dsdatascience.com/transfer-learning-with-convolutional-neuralnetworks-in-pytorch-dd09190245ce

29. Chilamkurthy S (2020) Transfer learning for computer vision tutorial
30. Kaggle (2013) Dogs vs. cats: create an algorithm to distinguish dogs from cats

31. R-Core-Team (2017) R Project. http://www.r-project.org

32. Runz M (2020). https://github.com/m4ln/pytorch_dcgan

33. Russakovsky O, Deng J, Su H, Krause J, Satheesh S, Ma S, Huang Z, Karpathy A, Khosla A, Bernstein M (2015) Imagenet large scale visual recognition challenge. Int J Comput Vis 115:211-252

34. Hosseini MS, Chan L, Tse G, Tang M, Deng J, Norouzi S, Rowsell C, Plataniotis KN, Damaskinos S (2019) Atlas of digital pathology: a generalized hierarchical histological tissue type-annotated database for deep learning, pp 11747-11756

35. Gayathri S, Gopi VP, Palanisamy P (2020) A lightweight CNN for diabetic retinopathy classification from fundus images. Biomed Signal Process Control 62:102115. https://doi.org/10.1016/j.bspc. 2020.102115

36. Echle A, Grabsch HI, Quirke P, van den Brandt PA, West NP, Hutchins GGA, Heij LR, Tan X, Richman SD, Krause J et al (2020) Clinical-grade detection of microsatellite instability in colorectal tumors by deep learning. Gastroenterology 159:14061416.e1411. https://doi.org/10.1053/j.gastro.2020.06.021

37. Kather JN, Pearson AT, Halama N, Jäger D, Krause J, Loosen SH, Marx A, Boor P, Tacke F, Neumann UP et al (2019) Deep learning can predict microsatellite instability directly from histology in gastrointestinal cancer. Nat Med 25:1054-1056. https://doi.org/ 10.1038/s41591-019-0462-y

Publisher's Note Springer Nature remains neutral with regard to jurisdictional claims in published maps and institutional affiliations. 